Marquette University

e-Publications@Marquette

7-28-2018

\title{
Spreading Electron Density Thin: Increasing the Chromophore Size in Polyaromatic Wires Decreases Interchromophoric Electronic Coupling
}

\author{
Mohammad Mosharraf Hossain \\ Marquette University \\ Maxim Vadimovich Ivanov \\ Marquette University \\ Denan Wang \\ Marquette University, denan.wang@marquette.edu \\ Scott A. Reid \\ Marquette University, scott.reid@marquette.edu \\ Rajendra Rathore \\ Marquette University, rajendra.rathore@marquette.edu
}

Follow this and additional works at: https://epublications.marquette.edu/chem_fac

Part of the Chemistry Commons

\section{Recommended Citation}

Hossain, Mohammad Mosharraf; Ivanov, Maxim Vadimovich; Wang, Denan; Reid, Scott A.; and Rathore, Rajendra, "Spreading Electron Density Thin: Increasing the Chromophore Size in Polyaromatic Wires Decreases Interchromophoric Electronic Coupling" (2018). Chemistry Faculty Research and Publications. 982.

https://epublications.marquette.edu/chem_fac/982 
Marquette University

e-Publications@Marquette

\section{Chemistry Faculty Research and Publications/College of Arts and Sciences}

This paper is NOT THE PUBLISHED VERSION; but the author's final, peer-reviewed manuscript. The published version may be accessed by following the link in th citation below.

Journal of Physical Chemistry C, Vol. 122, No. 31 (July 28, 2018): 17668-17678. DOI. This article is (C) American Chemical Society and permission has been granted for this version to appear in ePublications@Marquette. American Chemical Society does not grant permission for this article to be further copied/distributed or hosted elsewhere without the express permission from American Chemical Society.

\section{Spreading Electron Density Thin: Increasing the Chromophore Size in Polyaromatic Wires Decreases Interchromophoric Electronic Coupling}

\section{Mohammad M. Hossain}

Department of Chemistry, Marquette University, Milwaukee, Wisconsin

Maxim V. Ivanov

Department of Chemistry, Marquette University, Milwaukee, Wisconsin

Denan Wang

Department of Chemistry, Marquette University, Milwaukee, Wisconsin Scott A. Reid

Department of Chemistry, Marquette University, Milwaukee, Wisconsin Rajendra Rathore

Department of Chemistry, Marquette University, Milwaukee, Wisconsin 

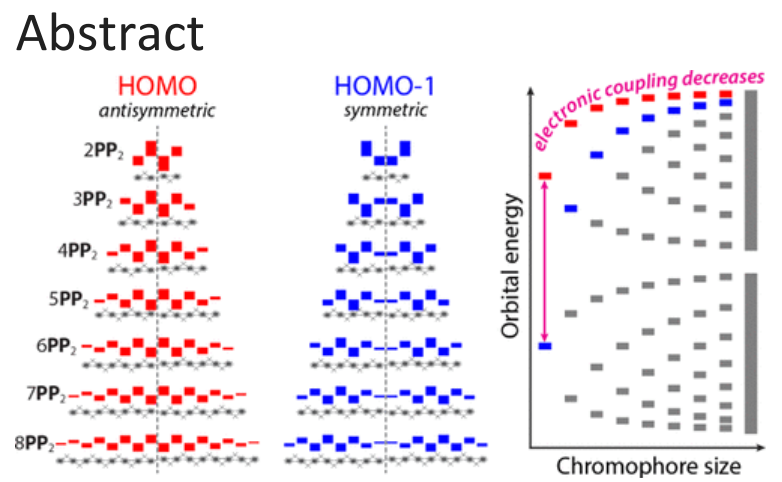

The development of novel polychromophoric materials using extended polycyclic aromatic hydrocarbons as a single large chromophore holds promise for long-range charge-transfer applications in photovoltaic devices and molecular electronics. However, it is not well-understood how the interchromophoric electronic coupling varies with the chromophore size in linearly connected molecular wires. Here, we show with the aid of electrochemistry, electronic spectroscopy, density functional theory calculations, and theoretical modeling that as the number of aromatic moieties in a single chromophore increases, the interchromophoric electronic coupling decreases and may reach negligible values if the chromophore is sufficiently large. The origin of this initially surprising result becomes clear when one considers this problem with the aid of Hückel molecular orbital theory, as at the polymeric limit energies of the molecular orbitals cluster to form bands and thus the energy spacing between orbitals, and thereby the electronic coupling must decrease with the chromophore expansion.

\section{Introduction}

Poly- $p$-phenylene-based wires are prototypical systems for charge-transfer studies with potential applications in photovoltaic and molecular electronics devices.(1-5) Electronic coupling between a pair of phenylenes in a poly$p$-phenylene-based wire is a crucial parameter that controls its redox and optical properties, as well as the rates of electron transfer in the corresponding donor-wire-acceptor systems.(6-9) For example, unsubstituted poly$p$-phenylene wires are characterized by strong interchromophoric electronic coupling because of the favorable nodal arrangement of the highest occupied molecular orbital (HOMO) lobes and relatively small interplanar dihedral angles, which promote effective orbital overlap between adjacent phenylenes.(10,11) This strong electronic coupling is reflected in the sensitivity of redox/optical properties to the wire length, as can be judged by large slopes in their $1 / n$ or $\cos [\pi /(n+1)]$ dependences, $(10,12)$ where $n$ is number of chromophoric units in a wire.

In this context, an interesting question concerns how many phenylenes should be included in a single chromophore. For example, a poly-p-phenylene-based wire shown in Figure 1 can be either considered as a poly-fluorene (i.e., $\mathbf{P F}_{n}$ ) or poly-p-phenylene (i.e., $\mathbf{P P}_{n}$ ). Irrespective of how the chromophore is defined, the absorption band of $\mathbf{P F}_{n}$ or $\mathbf{P P}_{n}$ shifts red with the increasing wire length, indicating that interchromophoric electronic coupling is significant (Figure 1).(10) However, can the value of the electronic coupling depend on how one defines the chromophore? 

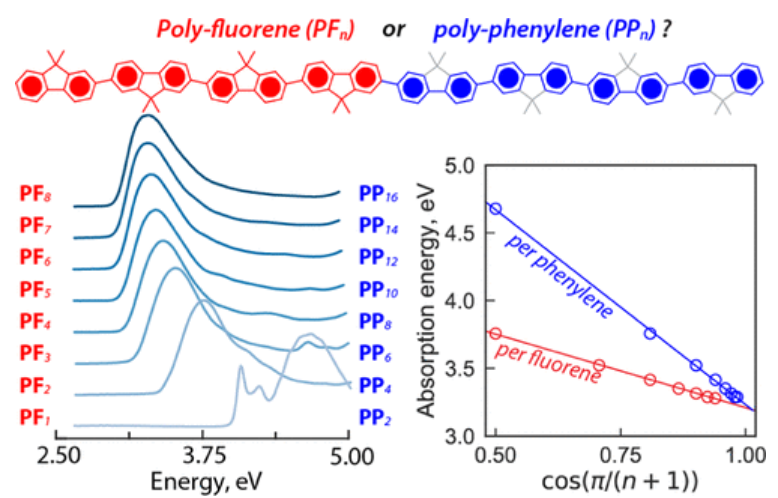

Figure 1. Depending on the choice of the monomeric unit, a molecular wire on the top can be either considered as poly-fluorene $\left(\mathbf{P F}_{n}\right)$ or poly-phenylene $\left(\mathbf{P P}_{n}\right)$. Left: Absorption spectra of $\mathbf{P} \mathbf{F}_{n}\left(\mathbf{o r} \mathbf{P P}_{n}\right)$. Right: Energies of maximum absorption plotted against $\cos [\pi /(n+1)]$, where $n$ is number of phenylenes (blue) or fluorenes (red).

According to the Hückel molecular orbital (MO) theory, the energy of the HOMO to LUMO transition scales linearly with $\cos [\pi /(n+1)]$, and the scaling factor depends on electronic coupling. $(10,13)$ Remarkably, the slope of the linear v-vs- $\cos [\pi /(n+1)]$ plot is smaller by nearly a factor of two when $n$ is number of fluorenes as compared to the plot where $n$ is the number of phenylenes (Figure 1), suggesting that the electronic coupling between fluorenes is by a factor of two smaller than that between a pair of phenylenes for the same wire.

As the magnitude of the electronic coupling directly impacts the redox/optical properties of the wire and the extent of hole (i.e., polaron) delocalization in the corresponding cation radicals, a choice of the chromophore size seems to be an important additional parameter. Indeed, a recent study showed on the example of biaryls with varied chromophore size that as the chromophore size increases, the mechanism of hole delocalization changes from static delocalization to dynamic hopping.(14) In order to systematically probe the curious dependence of the chromophore size on the electronic coupling, herein, we designed a set of poly-p-phenylenebased mono- and bichromophores (i.e., $n \mathbf{P P}$ and $n \mathbf{P P}_{2}$, Chart 1) where the size of the chromophore is varied from $n=2$ to 4 phenylenes. Aided by the electrochemical properties of $n \mathbf{P P} / n \mathbf{P P}_{2}$ and spectroscopic signatures of their cation radicals with the support from density functional theory (DFT) calculations, we conclude that as the size of the chromophore increases, the interchromophoric electronic coupling gradually decreases and may reach nearly nonexistent values at the polymeric limit. We show that the origin of this initially surprising result becomes clear when one considers this problem with the aid of Hückel MO theory, which predicts that at the polymeric limit, the energies of the MOs become nearly degenerate and the energy bands are formed, leading to the inevitability of the decrease of interchromophoric electronic coupling as the chromophore expands.

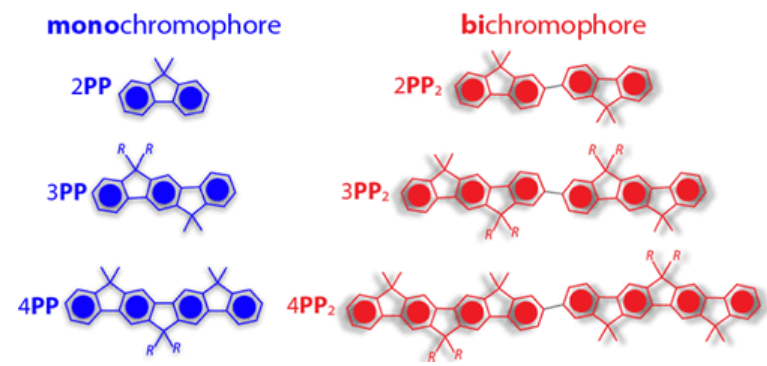

Chart 1. Structures of Monochromophoric $n \mathrm{PP}$ and Bichromophoric $n \mathbf{P P}_{2}(n=2-4, R=\mathrm{Hexyl})$ 


\section{Results and Discussion}

\section{Synthesis}

The preparation of monochromophores $n \mathbf{P P}$ and bichromophores $n \mathbf{P P}_{2}(n=3,4)$ was accomplished by adaptation of literature procedures $\underline{(15-23)}$ and involved alkylation of 9,9 position of fluorene unit, palladiumcatalyzed Suzuki coupling, alkylation of the carbonyl group of the ester to form the tertiary alcohol, and acidcatalyzed Friedel-Crafts intramolecular cyclization. The synthetic procedures are summarized in Scheme S1 in the Supporting Information along with the full experimental details. All compounds were characterized by ${ }^{1} \mathrm{H} /{ }^{13} \mathrm{C}$ NMR spectroscopy and matrix-assisted laser desorption ionization (MALDI) mass spectrometry.

\section{Electrochemistry}

The electron donor strengths of $n \mathbf{P P}$ and $n \mathbf{P P}_{2}(n=2-4)$ were evaluated by electrochemical oxidation at a platinum electrode as a $2 \mathrm{mM}$ solution in $\mathrm{CH}_{2} \mathrm{Cl}_{2}$ containing $0.1 \mathrm{M} \mathrm{n}-\mathrm{Bu}_{4} \mathrm{NPF}_{6}$ as the supporting electrolyte. The cyclic voltammograms (CVs) of $n P P$ showed that upon increasing the number of phenylenes, the first oxidation potential decreases from $E_{\text {ox }}=1.27 \mathrm{~V}$ vs Fc/Fc ${ }^{+}$in $2 \mathrm{PP}$ to $0.89 \mathrm{~V}$ in $3 \mathrm{PP}$ and to $0.68 \mathrm{~V}$ in $4 \mathrm{PP}$ (Figure 2).

Noteworthy, although 2PP (expectedly) displays an irreversible CV because of the presence of the substitutionlabile carbons that render the cation radicals susceptible to dimerization, the CVs of 3PP and 4PP were found to be completely reversible.
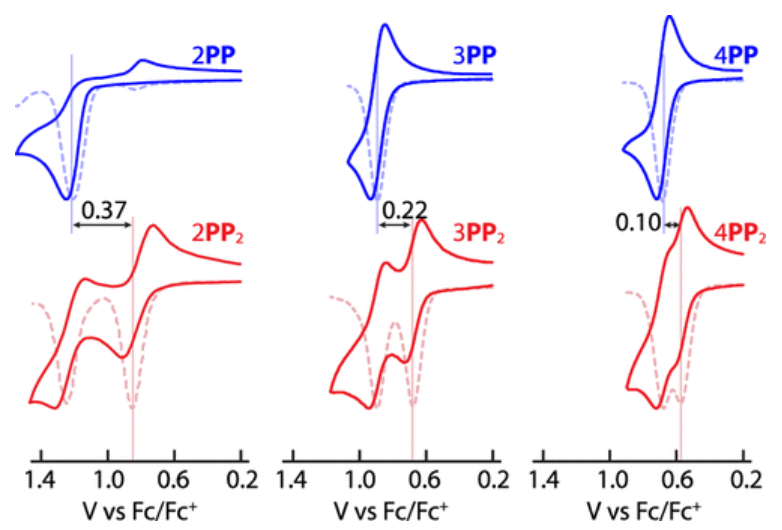

Figure 2. Cyclic (solid lines) and square-wave (dashed lines) voltammograms of $2 \mathrm{mM} n \mathbf{P P}$ (blue) and $n \mathbf{P P}_{2}$ (red) in $\mathrm{CH}_{2} \mathrm{Cl}_{2}\left(0.1 \mathrm{M} \mathrm{n}-\mathrm{Bu}_{4} \mathrm{NPF}_{6}\right)$ at $200 \mathrm{mV} \mathrm{s}^{-1}$ and $22{ }^{\circ} \mathrm{C}$. $\mathrm{CVs}$ and SWs of $2 \mathrm{PP}$ and $2 \mathrm{PP}_{2}$ are available from the literature.(10)

The reversible CVs of $n \mathbf{P P}_{2}(n=2-4)$ showed two well-separated oxidation waves that correspond to the formation of the cation radical and dication (Figure 2). As the number of phenylenes in each chromophore increases, the first oxidation potential of $n \mathbf{P P}_{2}$ decreases from $E_{\mathrm{ox}}=0.90 \mathrm{~V} \mathrm{vs} \mathrm{Fc} / F c^{+}$in $2 \mathbf{P P}_{2}$ to $0.67 \mathrm{~V}$ in $3 \mathbf{P P}_{2}$ and to $0.58 \mathrm{~V}$ in $4 \mathbf{P P}_{2}$. Because the $E_{\text {ox }}$ of monochromophoric $n \mathbf{P P}$ decreases faster with increasing $n$ than $E_{\text {ox }}$ of bichromophoric $n \mathbf{P P}_{2}$, the amount of hole stabilization, measured as the difference $\Delta E_{\mathrm{ox}}=E_{\mathrm{ox}}[n \mathbf{P P}]-E_{\mathrm{ox}}\left[n \mathbf{P P}_{2}\right]$, decreases from 0.37 to 0.22 to $0.10 \mathrm{~V}$, indicating that the interchromophoric electronic coupling decreases with increasing $n$ (Table1). Consistent with this conclusion, we also note that the separation between two oxidation waves in $n \mathbf{P P}_{2}$ decreases with increasing chromophore size, signifying that the repulsive interactions between the positive charge densities in $n \mathbf{P P}_{2}{ }^{2+}$ also decrease with increasing chromophore size.(24-27)

Table 1. Oxidation Potentials $\left(E_{\text {ox }}, \mathrm{V}\right.$ vs $\left.\mathrm{Fc} / \mathrm{Fc}^{+}\right)$of $n \mathrm{PP}$ and $n \mathrm{PP}_{2}$; Experimental $\left(\Delta E_{\text {ox, }} \mathrm{V}\right)$ and Computed $\left(\Delta G_{\text {ox }}, \mathrm{eV}\right)$ Using B1LYP40/6-31G(d) + PCM $\left(\mathrm{CH}_{2} \mathrm{Cl}_{2}\right)$ Hole Stabilizations; Experimental Wavelengths $(\lambda, \mathrm{nm})$ of Maximum Absorption of $n \mathrm{PP}^{*+}$ and $n \mathrm{PP}_{2}{ }^{*+} \underline{\mathrm{a}}$ 


\begin{tabular}{|l|l|l|l|l|l|l|}
\hline $\mathrm{n}$ & $\mathrm{E}_{\text {ox }}[\mathrm{nPP}]$ & $\mathrm{E}_{\mathrm{ox}}\left[n \mathrm{nP}_{2}\right]$ & $\Delta \mathrm{E}_{\text {ox }}$ & $\Delta \mathrm{G}_{\text {ox }}$ & $\lambda[\mathrm{nPP} \cdot+]$ & $\lambda\left[n \mathrm{nP}_{2}{ }^{++}\right]$ \\
\hline $\mathbf{2}$ & 1.27 & $\mathbf{0 . 9}$ & $\mathbf{0 . 3 7}$ & $\mathbf{0 . 3 7}$ & $\mathbf{6 9 0}$ & $\mathbf{1 2 4 0}$ \\
\hline $\mathbf{3}$ & $\mathbf{0 . 8 9}$ & $\mathbf{0 . 6 7}$ & $\mathbf{0 . 2 2}$ & $\mathbf{0 . 1 9}$ & $\mathbf{8 7 2}$ & $\mathbf{1 7 8 4}$ \\
\hline $\mathbf{4}$ & $\mathbf{0 . 6 8}$ & $\mathbf{0 . 5 8}$ & $\mathbf{0 . 1 0}$ & $\mathbf{0 . 0 8}$ & $\mathbf{1 1 0 0}$ & $\mathbf{2 3 3 0}$ \\
\hline
\end{tabular}

${ }^{2}$ Values of $\lambda\left[2 \mathrm{PP}^{*+}\right]$ and $\lambda\left[2 \mathrm{PP}_{2}{ }^{\circ+}\right]$ are available from literature.(10)

\section{Generation of Cation Radicals of $n P P$ and $n \mathrm{PP}_{2}$}

To further probe the electronic structure of $n \mathbf{P P}_{2}$, we resorted to electronic spectroscopy of their cation radicals. The cation radicals are expected to display an intervalence transition in the near-IR region, the position and intensity of which are sensitive to the degree of electronic coupling.(28-30) Cation radicals of $n \mathbf{P P} \mathbf{P}_{2}$ and $n \mathbf{P P}(n=$ 3 and 4$)$ were generated via quantitative $(31,32)$ redox titrations using two aromatic oxidants: [THEO ${ }^{*+} \mathrm{SbCl}_{6}{ }^{-}$] (THEO = tetrasubstituted $p$-hydroquinone ether, $E_{\text {red } 1}=0.67 \mathrm{~V} \mathrm{vs} \mathrm{Fc} / \mathrm{Fc}^{+}, \lambda_{\max }=518 \mathrm{~nm}$, and $\varepsilon_{\max }=7300 \mathrm{~cm}^{-1} \mathrm{M}^{-}$ $\left.{ }^{1}\right)(33)$ and $\left[\mathrm{NAP}^{\circ}+\mathrm{SbCl}_{6}^{-}\right.$] (NAP $=$cycloannulated naphthalene derivative, $E_{\text {red1 }}=0.94 \mathrm{~V} \mathrm{vs} \mathrm{Fc} / \mathrm{Fc}^{+}, \lambda_{\max }=672 \mathrm{~nm}$, and $\left.\varepsilon_{\max }=9300 \mathrm{~cm}^{-1} \mathrm{M}^{-1}\right) \cdot(34,35)$ Each redox titration experiment was carried out by an incremental addition of substoichiometric amounts of the electron donor (D, i.e., $n \mathbf{P P}_{2}$ or $\left.n \mathbf{P P}\right)$ to the solution of an oxidant cation

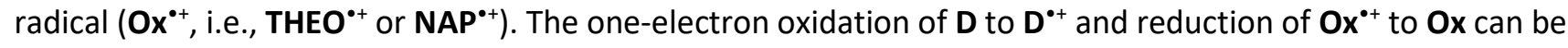
described by an equilibrium shown in eq 1.

$\mathrm{Ox}^{*+}+\mathrm{D} \rightleftharpoons \mathrm{Ox}+\mathrm{D}^{*+}(1)$

$$
\mathrm{Ox}^{\bullet+}+\mathrm{D} \rightleftarrows \mathrm{Ox}+\mathrm{D}^{\bullet+}
$$

The reproducible spectra of $n \mathbf{P P}^{\bullet+} / n \mathbf{P P}_{2}{ }^{\bullet+}$ were obtained by a numerical deconvolution procedure $(31,32)$ at each titration point that produced the mole fraction plots of each species involved in the redox reaction. The plots show that, in the case of $3 \mathbf{P P}, 4 \mathbf{P P}$, and $3 \mathbf{P P}_{2}$, the addition of a neutral donor leads to exclusive formation of the corresponding cation radical and complete consumption of the oxidant (Figure $3 A-F$ ). In the case of $4 \mathbf{P P}_{2}$, the redox titrations involved two successive one-electron oxidations with multiple equilibria (Figure $3 \mathrm{G}, \mathrm{H}$ ). Upon addition of 0.5 equiv of $4 \mathbf{P P}_{2}$, the oxidant is consumed completely and only the dication $4 \mathbf{P P}_{2}{ }^{2+}$ is formed because of similar values of its first and second oxidation energies (Table1). Upon further addition of 0.5 equivalents of neutral $4 \mathbf{P P}_{2}, 4 \mathbf{P P}_{2}{ }^{2+}$ is completely converted into the $4 \mathbf{P P}_{2}{ }^{{ }^{+}}$via a disproportionation reaction.(31)

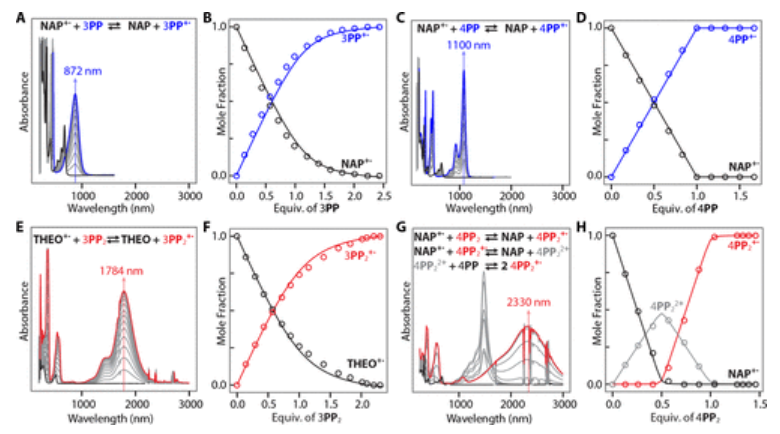

Figure 3. (A) Spectral changes observed upon the reduction of $0.036 \mathrm{mM} \mathrm{NAP \cdot +}$ in $\mathrm{CH}_{2} \mathrm{Cl}_{2}$ by incremental addition of $2.2 \mathrm{mM}$ solution of 3PP in $\mathrm{CH}_{2} \mathrm{Cl}_{2}$. (B) Mole fraction plot of NAP•+ (black) and 3PP•+ (blue) against the added equivalents of 3PP. Symbols represent experimental points, whereas the solid lines show best-fit to the experimental points using $\Delta G_{1}=-0.08 \mathrm{~V}$.(31) (C) Spectral changes observed upon the reduction of 0.021 mM NAP'+ in $\mathrm{CH}_{2} \mathrm{Cl}_{2}$ by incremental addition of $2.1 \mathrm{mM}$ solution of $4 \mathrm{PP}$ in $\mathrm{CH}_{2} \mathrm{Cl}_{2}$. (D) Mole fraction plot of NAP•+ (black) and 4PP•+ (blue) against the added equivalents of 4PP. Symbols represent experimental points, whereas the solid lines show best-fit to the experimental points using $\Delta G_{1}=-1.16 \mathrm{~V}$. (E) Spectral changes observed upon the reduction of $0.027 \mathrm{mM}$ THEO ${ }^{+}$in $\mathrm{CH}_{2} \mathrm{Cl}_{2}$ by incremental addition of $0.77 \mathrm{mM}$ solution of 
$3 \mathrm{PP}_{2}$ in $\mathrm{CH}_{2} \mathrm{Cl}_{2}$. (F) Mole fraction plot of THEO ${ }^{++}$(black) and $3 \mathrm{PP}_{2}{ }^{+}$(red) against the added equivalents of $3 \mathrm{PP}_{2}$. Symbols represent experimental points, whereas the solid lines show best-fit to the experimental points using $\Delta G_{1}=-0.06 \mathrm{~V}$. (G) Spectral changes observed upon the reduction of $0.018 \mathrm{mM} \mathrm{NAP*+}$ in $\mathrm{CH}_{2} \mathrm{Cl}_{2}$ by incremental addition of $0.45 \mathrm{mM}$ solution of $4 \mathbf{P P}_{2}$ in $\mathrm{CH}_{2} \mathrm{Cl}_{2}$. (H) Mole fraction plot of $\mathbf{N A P} \cdot+$ (black), $4 \mathbf{P P}_{2}{ }^{2+}$ (gray), and $4 \mathbf{P P}_{2}{ }^{\circ+}(\mathrm{red})$ against the added equivalents of $4 \mathbf{P P}_{2}$. Symbols represent experimental points, whereas the solid lines show best-fit to the experimental points using $\Delta G_{1}=-0.39 \mathrm{~V}$ and $\Delta G_{12}=0.22 \mathrm{mV}$.

Electronic absorption spectra of $n \mathbf{P P}_{2}{ }^{\bullet+}(n=3-4)$, obtained from the deconvolution, show the presence of a characteristic broad band that is red-shifted (i.e., shifted to longer wavelength) as compared to the corresponding monochromophoric $n \mathbf{P P}^{\bullet^{++}}$(年igure 3 ), signifying extensive hole delocalization in $n \mathbf{P P}_{2}{ }^{\bullet+}$. At the same time, as the number of phenylenes in the chromophore increases, the position of the near-IR band shifts to longer wavelengths, indicating that the electronic coupling is decreasing. To further probe the evolution of electronic coupling with the chromophore size, we resorted to DFT calculations.

\section{Electronic Structure Calculations}

Accurate description of the electronic structure of $\pi$-conjugated cation radicals is challenging for DFT because of the self-interaction error (SIE), which may lead in unfavorable cases to artificial hole delocalization, artificially low oxidation energies and an incorrect description of the excited states.(36-39) The SIE can be partially corrected by inclusion of a calibrated amount of the exact Hartree-Fock (HF) exchange term into the hybrid density functional. $(28,40)$ In our past studies, B1LYP(41) functional with $40 \%$ of HF exchange term (i.e., B1LYP40)(42) was introduced, where the amount of $\mathrm{HF}$ exchange term was fine-tuned to reproduce experimental oxidation potentials and cation radical excitation energies of poly-p-phenylenes with increasing number of $p$ phenylenes. Noteworthy, it was later shown that B1LYP-40/6-31G(d) performs exceptionally well in reproducing the experimental redox/optoelectronic properties of a variety of $\pi$-conjugated(10-12) and $\pi$-stacked assemblies(42-44) that were not included in the original training set. Therefore, in this manuscript, we performed DFT calculations of $n \mathrm{PP} / n \mathrm{PP}_{2}$ and their cation radical using the B1LYP-40/6-31G(d) method and account for the solvent effects using the polarizable continuum model (PCM) $(45)$ with $\mathrm{CH}_{2} \mathrm{Cl}_{2}$ parameters.

X-ray crystallography of numerous neutral aromatic hydrocarbons and their cation radicals has established that oxidation induces significant structural reorganization in the form of elongations and contractions of the $\mathrm{C}-\mathrm{C}$ bonds, as well as in the decrease of dihedral angles between adjacent aromatic moieties. $(46,47)$ Indeed, calculations showed that upon oxidation, the molecular structure of $n \mathbf{P P}_{2}$ undergoes a quinoidal distortion, as exemplified for $2 \mathbf{P P}_{2}$ in Figure $4 \mathrm{~A}$ below.

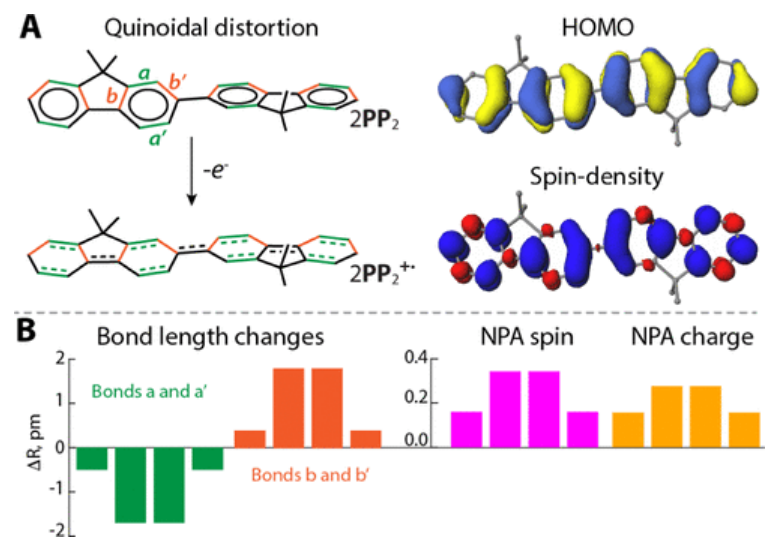


Figure 4. (A) Schematic representation of the quinoidal distortion in $2 \mathbf{P P}_{2} \rightarrow 2 \mathbf{P P}_{2}{ }^{\cdot+}$ transformation, $\mathrm{HOMO}$ of $2 \mathrm{PP}_{2}$, and spin density of $2 \mathrm{PP}_{2}{ }^{\cdot+}$ calculated using B1LYP-40/6-31G(d) $+\mathrm{PCM}\left(\mathrm{CH}_{2} \mathrm{Cl}_{2}\right)$. (B) Per-phenylene bar-plot representation of the distributions of the bond length changes, NPA spin, and charge distributions in $2 \mathbf{P P}_{2}{ }^{\circ}$.

Importantly, oxidation-induced bond length changes track in accordance with the disposition of the lobes of HOMO, that is, bonds that correspond to the antibonding lobes undergo contraction and bonds that correspond to the bonding lobes undergo elongations, for example, the average of bonds $a$ and $a^{\prime}$ contracts by $0.5-1.7 \mathrm{pm}$, whereas the average of bonds $b$ and $b^{\prime}$ elongates by 0.4-1.8 pm (Figure 4). The natural population analysis (NPA) of the electron density in $2 \mathbf{P P}_{2}{ }^{{ }^{++}}$further showed that the spin and charge distributions parallel the distribution of the bond-length changes, indicating polaron formation(48-50) (Figure 4B).

A simple yet intuitive model to describe the polaron (i.e., hole) delocalization in various mixed-valence compounds is the two-state Marcus-Hush theory, which predicts that the extent of polaron delocalization depends on the interplay between electronic coupling $\left(H_{\mathrm{ab}}\right)$ and reorganization energy $(\lambda) \cdot(28,29,51,52)$ In the large electronic coupling limit $\left(2 H_{a b} \geq \lambda\right)$, the polaron is evenly delocalized between two sites (i.e., class III or "static" delocalization), whereas in the limit of small electronic coupling $\left(2 H_{a b}<\lambda\right)$, the polaron is partially delocalized with the distribution maximum centered on one of the sites (i.e., class II or "dynamic" hopping). When electronic coupling is nonexistent $\left(H_{a b}=0\right.$, class $\left.\mathrm{I}\right)$, the polaron is fully localized on one site.

Partitioning the spin, charge, and structural reorganization distributions in $2 \mathbf{P P}_{2}{ }^{{ }^{++}}$between a pair of fluorenes rather than four phenylenes suggests that the polaron is evenly delocalized between the fluorenes, and hence the mechanism of polaron delocalization is "static" delocalization according to the two-state representation (Figure 5).

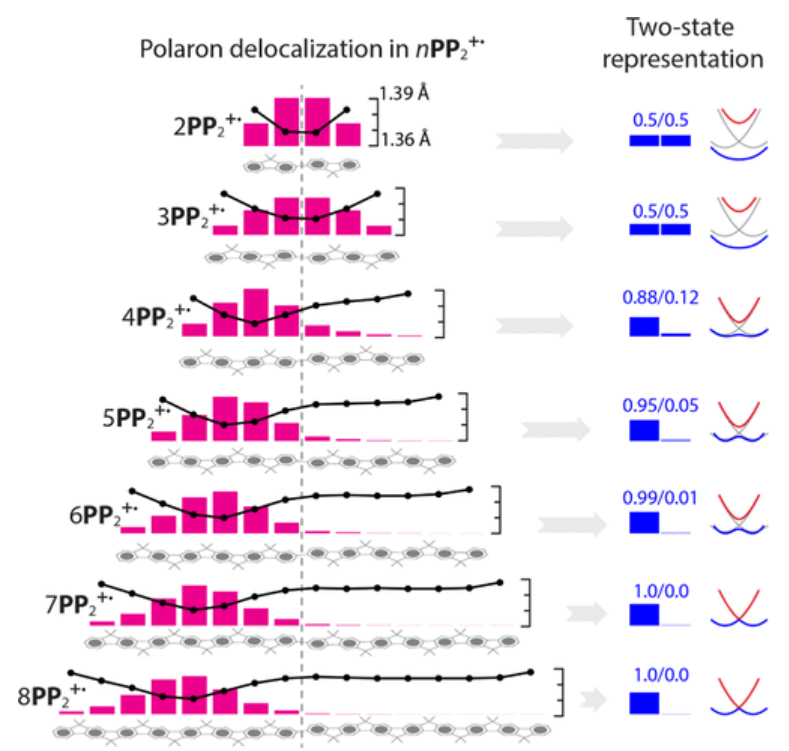

Figure 5. Left: Per-phenylene bar-plot representation of the distributions of the NPA spin (magenta) superimposed with the plot of $\mathrm{C}-\mathrm{C}$ bond length (average of $a$ and $a^{\prime}$, Figure 4 ) in $n \mathbf{P P}_{2}{ }^{\cdot+}$. Right: Two-state representation of polaron delocalization in $n \mathbf{P P}_{2}{ }^{\cdot+}$.

Similarly, in $3 \mathrm{PP}_{2}{ }^{{ }^{+}}$, the spin/charge/bond length changes are delocalized along the entire bichromophore, and thus the mechanism of polaron delocalization is also static delocalization. However, further increase of the chromophore size shifts the polaron distribution toward one side of $n \mathbf{P P}_{2}{ }^{\bullet+}(n>3)$, indicating that the mechanism of polaron delocalization evolves into dynamic hopping, that is, class II (igure 5 ). In the case of even 
longer $n \mathrm{PP}_{2}{ }^{\bullet+}(n>5)$, the polaron is fully localized on a single chromophore, that is, this bichromophore belongs to class I. Thus, depending on the chromophore size, the mechanism of hole delocalization in a two-state representation may switch from static delocalization to dynamic hopping to a complete localization, akin to the switchover observed for biaryls with varied interplanar angle.(52) For the latter, an increase in the interplanar angle led to the decrease in the overlap between p-orbitals of carbon atoms at the biaryl linkage, leading to the reduced electronic coupling. As the extent of hole delocalization depends on the interplay between electronic coupling $\left(H_{\mathrm{ab}}\right)$ and reorganization energy $(\lambda)$, the switchover in the delocalization mechanism in $n \mathbf{P P}_{2}{ }^{\bullet+}$ must arise because of the decreasing $H_{\mathrm{ab}}$ and/or increasing $\lambda$.(51)

To confirm that the switchover in the delocalization mechanism occurs because of the decreasing electronic coupling, we first compare computed oxidation free energies $\left(G_{o x}\right)$ of $n \mathbf{P P}$ and $n \mathbf{P P}_{2}(n=2-8)$. Calculations showed that as the number of phenylenes in the chromophore increases, oxidation energies of both $n \mathbf{P P}$ and $n \mathbf{P P}_{2}$ decrease (Figure $6 \mathrm{~A}$ ). Furthermore, hole stabilization, measured as the difference $\Delta G_{\text {ox }}=G_{\text {ox }}[n P P]-G_{\text {ox }}\left[n P_{2}\right]$, is the largest for $n=2$ and decreases with increasing $n$. Comparing the computed $\Delta G_{\text {ox }}$ with the available experimental values of $\Delta E_{\text {ox }}$ for $n=2-4$ (Table1), it is clear that the B1LYP-40 method shows a remarkable performance in reproducing hole stabilization for these systems, as the error in the computed $\Delta G_{\text {ox }}$ values is less than $0.03 \mathrm{eV}$. This suggests that calculations can provide reliable information on the extent of hole stabilization for the longer bichromophores, for which experimental data are unavailable. The calculations show that for longer $n \mathbf{P P}_{2}$ the increase in chromophore size reduces the hole stabilization to nearly nonexistent values, that is, $\Delta G_{o x}<0.01$ for $n>5$, which is consistent with complete localization of the polaron on a single chromophore.
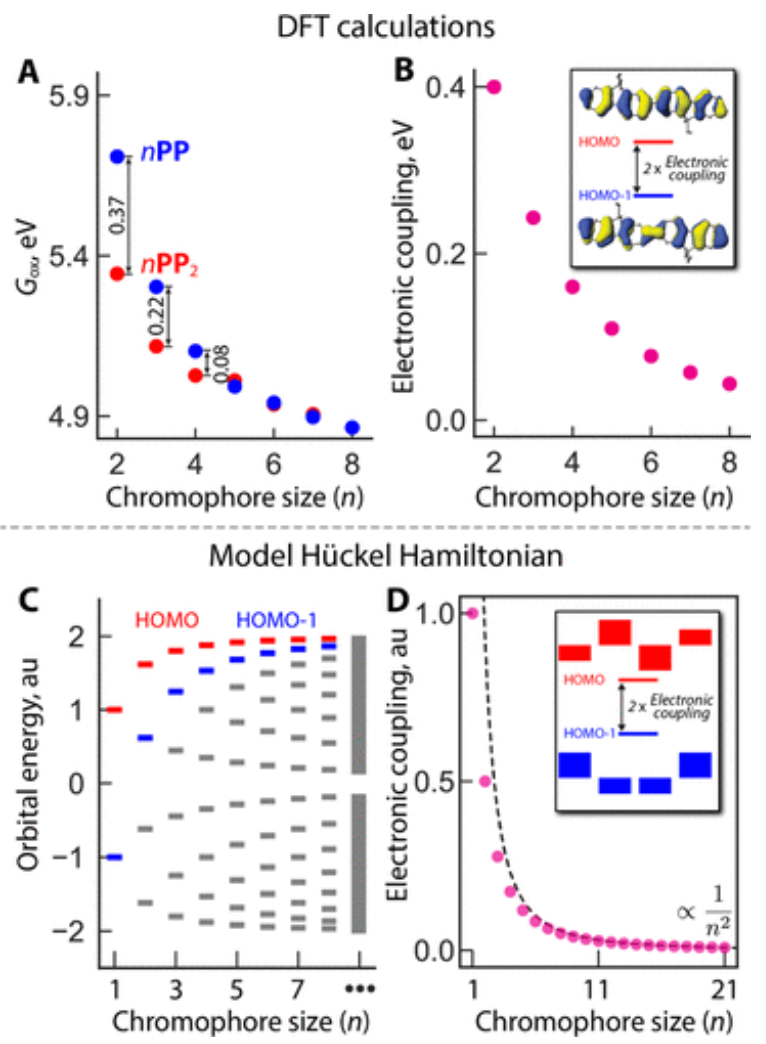

Figure 6. (A) Oxidation energies $\left(G_{o x}\right)$ of $n \mathbf{P P}$ (blue) and $n \mathbf{P P}_{2}$ (red) against the chromophore size measured in the number of phenylene units $(n)$ computed using B1LYP-40/6-31G(d) $+\mathrm{PCM}\left(\mathrm{CH}_{2} \mathrm{Cl}_{2}\right)$. (B) Interchromophoric electronic coupling of $n \mathbf{P P}_{2}$ measured as a half of the $\mathrm{HOMO} / \mathrm{HOMO}-1$ energy gap against chromophore size (n). Inset shows $\mathrm{HOMO}$ and $\mathrm{HOMO}-1$ of $2 \mathrm{PP}_{2}$. (C) Orbital energies calculated for a model Hückel Hamiltonian matrix (eq 2) against number of phenylene units in a chromophore. (D) Electronic coupling measured as a $\mathrm{HOMO} / \mathrm{HOMO}-1$ energy gap from a model Hückel Hamiltonian matrix against number of phenylene units in a 
chromophore. Dashed line corresponds to the $3 \pi^{2} / 8 n^{2}$ dependence derived in the limit of $n \rightarrow \infty$; see the Supporting Information for details. Inset shows per-phenylene bar-plot representation of HOMO and HOMO -1 wavefunctions of $2 \mathbf{P P}_{2}$ obtained from the Hückel Hamiltonian model.

\section{MO Theory of $n \mathrm{PP}_{2}$}

Following Hückel's approach of representing MOs as a linear combination of atomic orbitals, the two upper occupied frontier MOs of $n \mathbf{P P}_{2}$ obtained from DFT calculations can be approximated as symmetric and antisymmetric linear combinations of the HOMO of a single chromophore (Figure S5 in the Supporting Information). Then, the energy difference between $\mathrm{HOMO}$ and $\mathrm{HOMO}-1$ equals twice the electronic coupling and thus can be quickly determined from the DFT calculations of neutral $n \mathbf{P P}_{2}$. Following this approach, electronic couplings were computed for each $n \mathbf{P P}_{2}$, which showed a decrease with an increasing chromophore size (Figure 6B).

To provide an intuitive rationale for this observation, we resorted to a theoretical model. Following Hückel theory, each bichromophore $n \mathbf{P P}_{2}$ can be represented as a set of $2 n$ electronically coupled phenylenes with the Hamiltonian matrix $\mathbf{H}$ :

$\mathbf{H}=\left[\begin{array}{ccccc}\alpha & \beta & \cdots & 0 & 0 \\ \beta & \alpha & \cdots & 0 & 0 \\ \vdots & \vdots & \ddots & \vdots & \vdots \\ 0 & 0 & \cdots & \alpha & \beta \\ 0 & 0 & \cdots & \beta & \alpha\end{array}\right]_{(2)}$

$$
H=\left[\begin{array}{ccccc}
\alpha & \beta & \cdots & 0 & 0 \\
\beta & \alpha & \cdots & 0 & 0 \\
\vdots & \vdots & \ddots & \vdots & \vdots \\
0 & 0 & \cdots & \alpha & \beta \\
0 & 0 & \cdots & \beta & \alpha
\end{array}\right]
$$

where $\mathbf{H}$ is the $2 n \times 2 n$ tight-binding matrix, $\alpha$ is the orbital energy of a single phenylene, and $\beta$ is the electronic coupling between a pair of adjacent phenylenes. Note that analytical expressions of eigenvalues (i.e., MO energies) and eigenvectors (i.e., MO wavefunctions) of the Hückel Hamiltonian matrix are known from the original works. $(53,54)$ Here, for the sake of simplicity, we set $\alpha=0$ and $\beta=-1$.

Diagonalization of the Hamiltonian $\mathbf{H}$ for varied $n$ produced eigenvalues and eigenvectors, among which two largest eigenvalues correspond to the energies of $\mathrm{HOMO}$ and $\mathrm{HOMO}-1$ (Figure $6 \mathrm{C}$ ). The corresponding eigenvectors can be viewed as symmetric and antisymmetric linear combinations of the HOMO of a single chromophore (Figure 7). Thus, based on the simple Hückel Hamiltonian approach, the energy gap between HOMO and HOMO - 1 decreases with increasing number of phenylenes in a chromophore, leading to nearly isoenergetic values and thus negligible electronic coupling at large $n$ (Figure 6D). This is fully consistent with the results obtained from DFT calculations (igire $6 \mathrm{~B}$ ). In contrast to DFT calculations, solution to the Hückel Hamiltonian matrix is available in an analytic form $(53,54)$ and thus allows a derivation of the analytic formula for the electronic coupling, which in the limit of $n \rightarrow \infty$ follows a simple $3 \pi^{2} / 8 n^{2}$ dependence (see Figure 6D and the Supporting Information for details). 


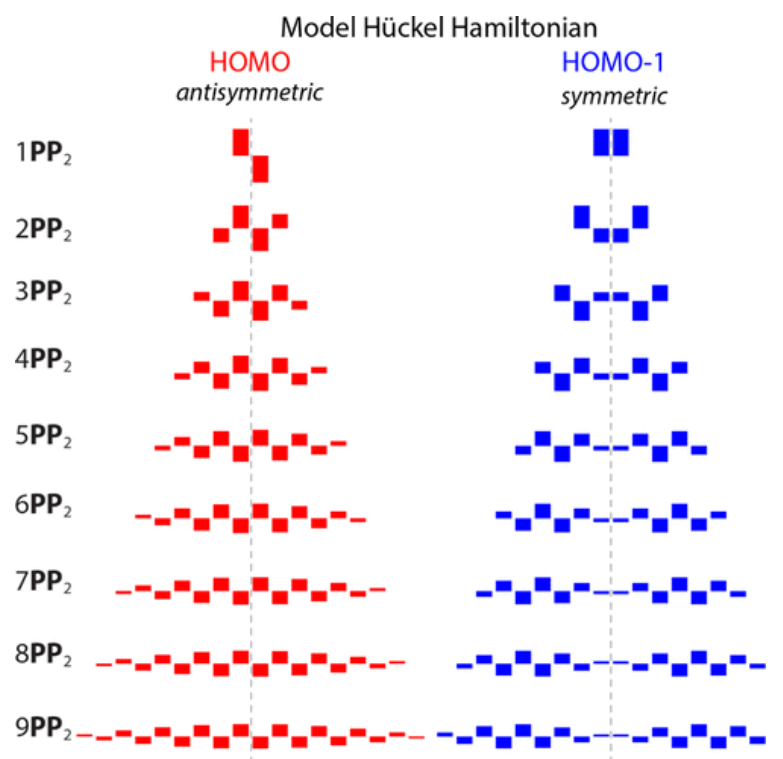

Figure 7. Bar-plot representations of the $\mathrm{HOMO}$ and $\mathrm{HOMO}-1$ wavefunctions of $n \mathbf{P P}_{2}$ obtained from the diagonalization of the Hückel Hamiltonian model.

We note that although in a discrete molecule, the MO energies are clearly distinguished; at the polymeric limit, the energy bands are formed that can be described by a continuous density of states (Figure 6B). $(55,56)$ It is thus inevitable that as the oligomer size increases, the spacing between its $\mathrm{MO}$ energies (HOMO and $\mathrm{HOMO}-1$ included) will decrease and reach near-degenerate values at the polymeric limit, which in the case of a bichromophoric oligomer can be interpreted as decreasing the electronic coupling between chromophores.

Following a recent study $(14)$ on the mechanism of hole delocalization in biaryls with varied donor strength of the substituent and varied size of the chromophore, an alternative explanation of the decreasing electronic coupling in $n \mathbf{P P}_{2}$ is warranted. This study showed that upon increase in the chromophore size, a total of two electrons per orbital spreads over a larger area, thus decreasing the amount of the electron density at the coupling-mediating carbons at the biaryl linkage and leading to the diminished orbital overlap and thereby diminished electronic coupling. Likewise, as the number of phenylenes in each chromophore of $n \mathbf{P P}_{2}$ increases, the electron density of HOMO spreads over a larger number of carbons, decreasing the electron density at the coupling-mediating carbons and thereby decreasing interchromophoric electronic coupling (Figure 7).

\section{Conclusions}

Motivated by the question whether a set of linearly connected fluorenes is better termed a poly-fluorene or poly-phenylene wire (Figure 1), we performed a combined experimental/theoretical study on a series of phenylene-based bichromophores $n \mathbf{P P}_{2}$ of increasing size. We found experimentally that as the number of phenylenes in a chromophore increases from $n=2$ to 4 , the amount of hole stabilization measured electrochemically decreases and the absorption band in the electronic spectra of $n \mathbf{P P}_{2}{ }^{{ }^{+}+}$shifts to longer wavelength, indicating that the interchromophoric electronic coupling decreases. Aided by the benchmarked DFT calculations of a series of long $n \mathrm{PP} / n \mathrm{PP}_{2}(n=2-8)$, we have shown that the electronic coupling decreases to nearly nonexistent values when $n>5$, leading to a complete localization of a polaron (i.e., hole) on a single chromophore in the corresponding $n \mathbf{P P}_{2}{ }^{\cdot+}$. Finally, using the Hückel Hamiltonian model, we showed that the decreasing electronic coupling is a consequence of the clustering of $\mathrm{MO}$ energy level as the length of the oligomer increases. This obvious, yet fundamental, understanding that the interchromophoric electronic coupling decreases with increasing chromophore size is crucial during the rational design of novel molecular 
wires with large chromophores, where long-range charge transfer can be mediated by incoherent hopping mechanisms.

\section{Supporting Information}

The Supporting Information is available free of charge on the ACS Publications website at DOI: $10.1021 /$ acs.jpcc.8b05299.

- Experimental and computational details and Hückel theory modeling (PDF)

- Equilibrium geometries of $n \mathbf{P P}$ and $n \mathbf{P P}_{2}(n=2-8)$ optimized at neutral and cation radical states (TXT)

- $p d f$

o jp8b05299 si 001.pdf (14.9 MB)

- text

o jp8b05299 si 002.txt (151.16 kb)

The authors declare no competing financial interest.

\section{Acknowledgments}

We thank the NSF (CHE-1508677) and NIH (R01-HL112639-04) for the financial support and Professor Marat Talipov for preliminary calculations. The calculations were performed on the high-performance computing cluster Père at Marquette University and XSEDE.

\section{References}

$\underline{1}$ Facchetti, A. П-conjugated Polymers for Organic Electronics and Photovoltaic Cell Applications. Chem. Mater. 2011, 23, 733- 758, DOI: 10.1021/cm102419z

$\underline{2}$ Li, C.; Liu, M.; Pschirer, N. G.; Baumgarten, M.; Müllen, K. Polyphenylene-based Materials for Organic Photovoltaics. Chem. Rev. 2010, 110, 6817-6855, DOI: 10.1021/cr100052z

$\underline{3}$ Goldschmidt, J. C.; Fischer, S. Upconversion for Photovoltaics-a Review of Materials, Devices and Concepts for Performance Enhancement. Adv. Opt. Mater. 2015, 3, 510- 535, DOI: 10.1002/adom.201500024

$\underline{4}$ Lambert, C. J. Basic Concepts of Quantum Interference and Electron Transport in Single-molecule Electronics. Chem. Soc. Rev. 2015, 44, 875-888, DOI: 10.1039/c4cs00203b

$\underline{5}$ Holliday, S.; Li, Y.; Luscombe, C. K. Recent Advances in High Performance Donor-acceptor Polymers for Organic Photovoltaics. Prog. Polym. Sci. 2017, 70, 34- 51, DOI: 10.1016/j.progpolymsci.2017.03.003

$\underline{6}$ Li, G.; Govind, N.; Ratner, M. A.; Cramer, C. J.; Gagliardi, L. Influence of Coherent Tunneling and Incoherent Hopping on the Charge Transfer Mechanism in Linear Donor-Bridge-Acceptor Systems. J. Phys. Chem. Lett. 2015, 6, 4889-4897, DOI: 10.1021/acs.jpclett.5b02154

7 Migliore, A. Nonorthogonality Problem and Effective Electronic Coupling Calculation: Application to Charge Transfer in $\Pi$-Stacks Relevant to Biochemistry and Molecular Electronics. J. Chem. Theory Comput. 2011, 7, 1712- 1725, DOI: 10.1021/ct200192d

$\underline{8}$ Kawatsu, T.; Coropceanu, V.; Ye, A.; Brédas, J.-L. Quantum-chemical Approach to Electronic Coupling: Application to Charge Separation and Charge Recombination Pathways in a Model Molecular Donor- 
Acceptor System for Organic Solar Cells. J. Phys. Chem. C 2008, 112, 3429- 3433, DOI: 10.1021/jp711186j

9 Paddon-Row, M. N.; Shephard, M. J. Through-bond Orbital Coupling, the Parity Rule, and the Design of Superbridges Which Exhibit Greatly Enhanced Electronic Coupling: A Natural Bond Orbital Analysis. J. Am. Chem. Soc. 1997, 119, 5355- 5365, DOI: 10.1021/ja964132x

10 Ivanov, M. V.; Talipov, M. R.; Boddeda, A.; Abdelwahed, S. H.; Rathore, R. Hückel Theory+ Reorganization Energy $=$ Marcus-Hush TheoryBreakdown of the $1 / n$ Trend in $\Pi$-Conjugated Poly-p-phenylene Cation Radicals Is Explained. J. Phys. Chem. C 2017, 121, 1552- 1561, DOI: 10.1021/acs.jpcc.6b12111

$\underline{11}$ Ivanov, M. V.; Chebny, V. J.; Talipov, M. R.; Rathore, R. Poly-p-hydroquinone Ethers: Isoenergetic Molecular Wires with Length-Invariant Oxidation Potentials and Cation Radical Excitation Energies. J. Am. Chem. Soc. 2017, 139, 4334- 4337, DOI: 10.1021/jacs.7b01226

12 Wang, D.; Talipov, M. R.; Ivanov, M. V.; Rathore, R. Energy Gap Between the Poly-p-phenylene Bridge and Donor Groups Controls the Hole Delocalization in Donor-Bridge-Donor Wires. J. Am. Chem. Soc. 2016, 138, 16337-16344, DOI: 10.1021/jacs.6b09209

13 Larsen, R. E. Simple Extrapolation Method to Predict the Electronic Structure of Conjugated Polymers From Calculations on Oligomers. J. Phys. Chem. C 2016, 120, 9650-9660, DOI: 10.1021/acs.jpcc.6b02138

14 Ivanov, M. V.; Wang, D.; Rathore, R. From Static to Dynamic: Electron Density of HOMO at Biaryl Linkage Controls the Mechanism of Hole Delocalization. J. Am. Chem. Soc. 2018, 140, 4765- 4769, DOI: 10.1021/jacs.8b00466

15 Huang, H.-H.; Prabhakar, C.; Tang, K.-C.; Chou, P.-T.; Huang, G.-J.; Yang, J.-S. Ortho-branched Ladder-type Oligophenylenes with Two-dimensionally ח-conjugated Electronic Properties. J. Am. Chem. Soc. 2011, 133, 8028- 8039, DOI: 10.1021/ja202144v

16 Yang, C.; Mehmood, F.; Lam, T. L.; Chan, S. L.-F.; Wu, Y.; Yeung, C.-S.; Guan, X.; Li, K.; Chung, C. Y.-S.; Zhou, C.Y. Stable Luminescent Iridium (iii) Complexes with Bis (N-heterocyclic Carbene) Ligands: Photo-stability, Excited State Properties, Visible-light-driven Radical Cyclization and $\mathrm{CO} 2$ Reduction, and Cellular Imaging. Chem. Sci. 2016, 7, 3123-3136, DOI: 10.1039/c5sc04458h

17 Yang, J.; Jiang, C.; Zhang, Y.; Yang, R.; Yang, W.; Hou, Q.; Cao, Y. High-efficiency Saturated Red Emitting Polymers Derived From Fluorene and Naphthoselenadiazole. Macromolecules 2004, 37, 1211-1218, DOI: 10.1021/ma035743u

18 Jin, J.-K.; Choi, J.-K.; Kim, B.-J.; Kang, H.-B.; Yoon, S.-C.; You, H.; Jung, H.-T. Synthesis and Photovoltaic Performance of Low-bandgap Polymers on the Basis of 9, 9-dialkyl-3, 6dialkyloxysilafluorene. Macromolecules 2011, 44, 502- 511, DOI: 10.1021/ma102173a

19 Onitsuka, K.; Fujimoto, M.; Kitajima, H.; Ohshiro, N.; Takei, F.; Takahashi, S. Convergent Synthesis of Platinum-acetylide Dendrimers. Chem. -Eur. J. 2004, 10, 6433-6446, DOI: 10.1002/chem.200400544

20 Dudek, S. P.; Pouderoijen, M.; Abbel, R.; Schenning, A. P. H. J.; Meijer, E. W. Synthesis and Energy-transfer Properties of Hydrogen-bonded Oligofluorenes. J. Am. Chem. Soc. 2005, 127, 11763-11768, DOI: 10.1021/ja052054k

$\underline{21}$ Parsons, W. H.; Calvo, R. R.; Cheung, W.; Lee, Y.-K.; Patel, S.; Liu, J.; Youngman, M. A.; Dax, S. L.; Stone, D.; Qin, N. Benzo [d] Imidazole Transient Receptor Potential Vanilloid 1 Antagonists for the Treatment of Pain: Discovery of Trans-2-(2-2-[2-(4-Trifluoromethyl-phenyl)-vinyl]-1 H-benzimidazol-5-yl\}-phenyl)propan-2-ol (Mavatrep). J. Med. Chem. 2015, 58, 3859- 3874, DOI: 10.1021/acs.jmedchem.5b00132

$\underline{22}$ Elmahdy, M. M.; Floudas, G.; Oldridge, L.; Grimsdale, A. C.; Müllen, K. Self-Assembly and Molecular Dynamics of Oligoindenofluorenes. ChemPhysChem 2006, 7, 1431-1441, DOI: 10.1002/cphc.200600015

$\underline{23}$ Kobin, B.; Grubert, L.; Blumstengel, S.; Henneberger, F.; Hecht, S. Vacuum-processable Ladder-type Oligophenylenes for Organic-inorganic Hybrid Structures: Synthesis, Optical and Electrochemical Properties Upon Increasing Planarization As Well As Thin Film Growth. J. Mater.

Chem. 2012, 22, 4383, DOI: 10.1039/c2jm15868j 
$\underline{24}$ Ivanov, M. V.; Wadumethrige, S. H.; Wang, D.; Rathore, R. Unraveling the Coulombic Forces in Electronically Decoupled Bichromophoric Systems During Two Successive Electron Transfers. Chem.-Eur. J. 2017, 23, 8834-8838, DOI: 10.1002/chem.201702211

25 Winter, R. F. Half-Wave Potential Splittings $\Delta$ E $1 / 2$ As a Measure of Electronic Coupling in Mixed-Valent Systems: Triumphs and Defeats. Organometallics 2014, 33, 4517-4536, DOI: 10.1021/om500029x

$\underline{26}$ Geiger, W. E.; Barrière, F. Organometallic Electrochemistry Based on Electrolytes Containing Weaklycoordinating Fluoroarylborate Anions. Acc. Chem. Res. 2010, 43, 1030-1039, DOI: 10.1021/ar1000023

27 Chi, C.; Wegner, G. Chain-Length Dependence of the Electrochemical Properties of Conjugated Oligofluorenes. Macromol. Rapid Commun. 2005, 26, 1532-1537, DOI: 10.1002/marc.200500437

$\underline{28}$ Kaupp, M.; Renz, M.; Parthey, M.; Stolte, M.; Würthner, F.; Lambert, C. Computational and Spectroscopic Studies of Organic Mixed-valence Compounds: Where Is the Charge?. Phys. Chem. Chem. Phys. 2011, 13, 16973-16986, DOI: 10.1039/c1cp21772k

$\underline{29}$ Heckmann, A.; Lambert, C. Organic Mixed-Valence Compounds: A Playground for Electrons and Holes. Angew. Chem., Int. Ed. 2012, 51, 326-392, DOI: 10.1002/anie.201100944

30 Nelsen, S. F. Almost Delocalized Intervalence Compounds. Chem. -Eur. J. 2000, 6, 581- 588, DOI: 10.1002/(sici)1521-3765(20000218)6:4<581::aid-chem581>3.0.co;2-e

31 Talipov, M. R.; Boddeda, A.; Hossain, M. M.; Rathore, R. Quantitative Generation of Cation Radicals and Dications Using Aromatic Oxidants: Effect of Added Electrolyte on the Redox Potentials of Aromatic Electron Donors. J. Phys. Org. Chem. 2015, 29, 227- 233, DOI: 10.1002/poc.3523

32 Talipov, M. R.; Hossain, M. M.; Boddeda, A.; Thakur, K.; Rathore, R. A Search for Blues Brothers: X-ray Crystallographic/spectroscopic Characterization of the Tetraarylbenzidine Cation Radical As a Product of Aging of Solid Magic Blue. Org. Biomol. Chem. 2016, 14, 2961-2968, DOI: 10.1039/c6ob00140h

33 Rathore, R.; Burns, C. L.; Deselnicu, M. I. Preparation of 1, 4: 5, 8-Dimethano-1, 2, 3, 4, 5, 6, 7, 8-Octahydro-9, 10-Dimethoxyanthracenium Hexachloroantimonate (4+ SbCl6-): A Highly Robust Radical-Cation Salt. Org. Synth. 2005, 1-9, DOI: 10.1002/0471264229.0s082.01

34 Rathore, R.; Burns, C. L.; Deselnicu, M. I. Multiple-electron Transfer in a Single Step. Design and Synthesis of Highly Charged Cation-radical Salts. Org. Lett. 2001, 3, 2887-2890, DOI: 10.1021/ol0163474

$\underline{35}$ Rathore, R.; Kochi, J. K. Acid Catalysis Vs. Electron-transfer Catalysis Via Organic Cations or Cation-radicals As the Reactive Intermediate. Are These Distinctive Mechanisms?. Acta Chem.

Scand. 1998, 52, 114- 130, DOI: 10.3891/acta.chem.scand.52-0114

36 Cohen, A. J.; Mori-Sánchez, P.; Yang, W. Challenges for Density Functional Theory. Chem. Rev. 2012, 112, 289-320, DOI: 10.1021/cr200107z

37 Ranasinghe, D. S.; Margraf, J. T.; Jin, Y.; Bartlett, R. J. Does the lonization Potential Condition Employed in QTP Functionals Mitigate the Self-interaction Error?. J. Chem. Phys. 2017, 146, 034102, DOI: 10.1063/1.4973727

38 Lundberg, M.; Siegbahn, P. E. M. Quantifying the Effects of the Self-interaction Error in DFT: When Do the Delocalized States Appear?. J. Chem. Phys. 2005, 122, 224103, DOI: 10.1063/1.1926277

39 Zhang, Y.; Yang, W. A Challenge for Density Functionals: Self-interaction Error Increases for Systems with a Noninteger Number of Electrons. J. Chem. Phys. 1998, 109, 2604- 2608, DOI: 10.1063/1.476859

40 Félix, M.; Voityuk, A. A. DFT Performance for the Hole Transfer Parameters in DNA $\Pi$ Stacks. Int. J. Quantum Chem. 2011, 111, 191-201, DOI: 10.1002/qua.22419

41 Adamo, C.; Barone, V. Toward Reliable Adiabatic Connection Models Free From Adjustable Parameters. Chem. Phys. Lett. 1997, 274, 242-250, DOI: 10.1016/s0009-2614(97)00651-9

42 Talipov, M. R.; Boddeda, A.; Timerghazin, Q. K.; Rathore, R. Key Role of End-capping Groups in Optoelectronic Properties of Poly-p-phenylene Cation Radicals. J. Phys. Chem. C 2014, 118, 21400- 21408, DOI: 10.1021/jp5082752 
43 Ivanov, M. V.; Reilly, N.; Uhler, B.; Kokkin, D.; Rathore, R.; Reid, S. A. Cofacially-Arrayed Polyfluorenes: Spontaneous Formation of $\Pi$-Stacked Assemblies in the Gas-Phase. J. Phys. Chem.

Lett. 2017, 8, 5272- 5276, DOI: 10.1021/acs.jpclett.7b02627

$\underline{44}$ Kokkin, D.; Ivanov, M. V.; Loman, J.; Cai, J.-Z.; Rathore, R.; Reid, S. A. Strength of ח-Stacking, From Neutral to Cation: Precision Measurement of Binding Energies in An Isolated ח-Stacked Dimer. J. Phys. Chem. Lett. 2018, 9, 2058- 2061, DOI: 10.1021/acs.jpclett.8b00742

45 Cancès, E.; Mennucci, B.; Tomasi, J. A New Integral Equation Formalism for the Polarizable Continuum Model: Theoretical Background and Applications to Isotropic and Anisotropic Dielectrics. J. Chem. Phys. 1997, 107, 3032, DOI: 10.1063/1.474659

46 Ivanova, L. V.; Wang, D.; Lindeman, S.; Ivanov, M. V.; Rathore, R. Probing Charge Delocalization in Solid State Polychromophoric Cation Radicals Using X-ray Crystallography and DFT Calculations. J. Phys. Chem. C 2018, 122, 9339- 9345, DOI: 10.1021/acs.jpcc.8b02184

47 Navale, T. S.; Thakur, K.; Vyas, V. S.; Wadumethrige, S. H.; Shukla, R.; Lindeman, S. V.; Rathore, R. Charge Delocalization in Self-assembled Mixed-valence Aromatic Cation Radicals. Langmuir 2012, 28, 71- 83, DOI: 10.1021/la202611w

48 Donati, G.; Lingerfelt, D. B.; Petrone, A.; Rega, N.; Li, X. "Watching” Polaron Pair Formation From FirstPrinciples Electron-Nuclear Dynamics. J. Phys. Chem. A 2016, 120, 7255- 7261, DOI: 10.1021/acs.jpca.6b06419

49 Nayyar, I. H.; Batista, E. R.; Tretiak, S.; Saxena, A.; Smith, D. L.; Martin, R. L. Role of Geometric Distortion and Polarization in Localizing Electronic Excitations in Conjugated Polymers. J. Chem. Theory Comput. 2013, 9, 1144-1154, DOI: 10.1021/ct300837d

$\underline{50}$ Neukirch, A. J.; Nie, W.; Blancon, J.-C.; Appavoo, K.; Tsai, H.; Sfeir, M. Y.; Katan, C.; Pedesseau, L.; Even, J.; Crochet, J. J.Polaron Stabilization by Cooperative Lattice Distortion and Cation Rotations in Hybrid Perovskite Materials. Nano Lett. 2016, 16, 3809-3816, DOI: 10.1021/acs.nanolett.6b01218

$\underline{51}$ Brunschwig, B. S.; Creutz, C.; Sutin, N. Optical Transitions of Symmetrical Mixed-valence Systems in the Class II-III Transition Regime. Chem. Soc. Rev. 2002, 31, 168-184, DOI: 10.1039/b008034i

$\underline{\mathbf{5 2}}$ Talipov, M. R.; Navale, T. S.; Hossain, M. M.; Shukla, R.; Ivanov, M. V.; Rathore, R. Dihedral Angle-Controlled Crossover From Static Hole Delocalization to Dynamic Hopping in Biaryl Cation Radicals. Angew. Chem., Int. Ed. 2017, 56, 266-269, DOI: 10.1002/anie.201609695

$\underline{53}$ Hückel, E. Quantentheoretische Beiträge Zum Problem Der Aromatischen Und Ungesättigten Verbindungen. III. Z. Phys. 1932, 76, 628-648, DOI: 10.1007/bf01341936

54 Frost, A. A.; Musulin, B. A Mnemonic Device for Molecular Orbital Energies. J. Chem. Phys. 1953, 21, 572- 573, DOI: 10.1063/1.1698970

55 Hoffmann, R. How Chemistry and Physics Meet in the Solid State. Angew. Chem., Int. Ed. 1987, 26, 846- 878, DOI: 10.1002/anie.198708461

$\underline{56}$ Kashimoto, Y.; Yonezawa, K.; Meissner, M.; Gruenewald, M.; Ueba, T.; Kera, S.; Forker, R.; Fritz, T.; Yoshida, $\mathrm{H}$. The Evolution of Intermolecular Energy Bands of Occupied and Unoccupied Molecular States in Organic Thin Films. J. Phys. Chem. C 2018, 122, 12090- 12097, DOI: 10.1021/acs.jpcc.8b02581 